\title{
ANGIOMYOFIBROBLASTOMA OF VULVA: A CASE REPORT
}

R. C. Prameela ${ }^{1}$, Anusha K. S², Akhila M. V. ${ }^{3}$

\section{HOW TO CITE THIS ARTICLE:}

R. C. Prameela ${ }^{1}$, Anusha K. S ${ }^{2}$, Akhila M. V. "Angiomyo Fibro Blastoma of Vulva: A Case Report". Journal of Evolution of Medical and Dental Sciences 2014; Vol. 3, Issue 45, September 18; Page: 11130-11133,

DOI: $10.14260 /$ jemds/2014/3452

ABSTRACT: Angiomyofibroblastoma (AMF) is a rare soft tissue tumor with unique morphological features occurring in the vulvo vaginal region of women. Few reported cases of occurrence in men. The tumor is localized in the scrotum \&in the inguinal area of male patients. It is often mistaken for Bartholin's gland cyst in women. We report a case of Angiomyofibroblastoma of vulva in a 46 yrs old woman.

INTRODUCTION: Vulval tumors are rare, ${ }^{1,2}$ usually distributed among Dermatology, Genito urinary medicine, Gynecology and Pathology. Due to complexity of vulvar diseases there is a necessity of multi-disciplinary approach. ${ }^{3}$ Angiomyofibroblastoma is a rare benign mesenchymal tumor occurring in middle aged women. ${ }^{4}$ Clinically it is misdiagnosed as a Bartholin's Gland Cyst. It is also reported in men in scrotal \& inguinalregion. ${ }^{5}$ We report a case of angiomyofibroblastoma in a $46 \mathrm{yr}$. old women in left labia majora.

CASE PRESENTATION: A 46 yrs old woman was admitted to our hospital with a complaint of swelling in the vulval region for 2 months \& pain of burning type in the swelling for 1 month. She noticed initially as a small painless swelling, it went on to increase to the present size. There was no history of fever, no history of vaginal discharge. Woman was moderately built, other systems normal clinically.

Local inspection a tense swelling of $10 x 8 \mathrm{~cm}$ seen in the left labia majora, extending medially to $1 / 3$ of vagina, laterally involving labia majora, superiorly up to urethral meatus, inferiorly upto fourchette. There was deviation of urethral opening. (Fig. 1) On palpation swelling was non-tender firm in consistency Extent of swellings were confirmed. Inguinal lymph nodes not palpable. Vaginal examination normal.

Serological, hematological investigations were within normal limits. Clinical diagnosis of soft tissue tumor of vulva was made and differential diagnosis of Bartholin's gland cyst was kept in mind. Decided for excision of mass. Under spinal anesthesia excision of the mass was done. Per operative finding the tumor was in the sub cutaneous tissue size $8 \times 10 \mathrm{~cm}$, non-encapsulated, lobulated surface, non-vascular. Firm in consistency located in left labia majora. Mass weighed 100 gms (Fig. 2).

Cut section revealed fibro fatty tissue with multiple blood filled cystic spaces. (Fig. 3)

HISTOPATHOLOGICAL EXAMINATION: Multiple sections studied showed oval spindle cells arranged in fascicles with alternating hypercellular and hypocellular areas admixed with small blood vessels. These spindle cells arranged in fascicles with alternating cells have indistinct cell borders with eosinophilic cytoplasm, oval to spindle nuclei with vesicular chromatin. Stroma shows minimal mucin deposition and occasional mitosis. 
IMPRESSION: Angiomyofibroblastoma (AMF).

DISCUSSION: This distinctive tumor Angiomyofibroblastoma (AMF) was first delineated by Fletcher et al. 1992 described only10 cases. ${ }^{1}$ There is a marked predilection for female genital tract although rare cases have been reported in scrotum and inguinal region of males. ${ }^{1}$ Regarding the female patients, it is believed that the tumor is derived from mesenchymal cells in the sub epithelial myxoid stromal zone that extends from the endo cervix to the vulva ${ }^{2}$ this hypothesis explaining to some extent the propensity of this tumor to arise in the lower genital tract.

The cell of origin of Angiomyofibroblastoma (AMF) among males has not been identified with certainty. All the published studies of patients with AMF have presented as benign tumours without local recurrence or metastatic potential. However latter possibility cannot be excluded since the respective follow up period was relatively small. The most crucial issue is to determine whether this case should be assigned to AMF or to aggressive angiomyxoma (AAM).

The latter is a histologically benign soft tissue tumor, associated with a high risk of local recurrence as well as with local infiltration that often results in entrapment of nerves and mucosal glands, thus making complete excision difficult. It has been suggested that AMF and AAM are related neoplasms, both included in a wide spectrum of angiomyxoid tumors, which exhibit some overlapping features and various combinations of myofibroblastic, fibroblastic and lipomatous differentiation.

The macroscopic characteristics of our case report are in agreement with those demonstrated by AMF rather than AAM, since the tumor was a well-circumscribed, relatively small-sized lesion with no infiltrating margins. ${ }^{5}$ Furthermore, the recognition of cytologic features reminiscent of myofibroblastic differentiation is paramount for the diagnosis of genital AMF and its differentiation from AAM. The immune-phenotype of angiomyofibroblastoma AMF is not distinct but most cases are desmin positive and $\alpha$ smooth muscle actin negative. However, some are negative for desmin or positive for $\alpha$ smooth muscle actin.

In case of AAM there was focal strong immunoreactivity for desmin with only occasional cells staining with anti-alpha smooth muscle actions. Angiomyofibroblastoma AMF is diffusely positive for ER and PR (estrogen \& progesterone receptors) and this has been described previously in vulval angiomyofibroblastoma. ${ }^{5}$ Although this raises the possibility that angiomyofibroblastoma is a hormone responsive neoplasm, positivity for ER and PR might simply be a reflection of the presence of these receptors normally in the sub epithelial stromal cells of the vulva and vagina.

It is probable that angiomyofibroblastoma (AMF) in this region is derived from mesenchymal cells in the sub epithelial myxoid stromal zone, which extends from the endocervix to the vulva. The differential diagnosis of AMF includes Bartholin's gland cyst, as in our case, smooth muscle tumors, peripheral nerve sheath tumors, glomus tumor, chondroid syringoma, myxoid malignant fibrous histiocytoma, angiomyolipoma, spindle cell lipoma and myxoid liposarcoma.

As most of these diagnoses were introduced before the original description of AMF, the aforementioned entities should be easily discriminated from AMF by routine light microscopic examination in conjunction with immune histochemical studies and electron microscopic examination in selected cases. ${ }^{2}$ Regarding the surgical management of these rare tumors (AMF), the most important factor for prognosis is the surgical and macroscopic delimitation of the tumor. 
Because most AMFs have been successfully treated with simple excision, this seems to be the appropriate therapy for these tumors.

\section{REFERENCES:}

1. Fletcher CD, Tsang WY, Fisher C, Lee KC, Chan JK. Angiomyofibroblastoma of the vulva. A benign neoplasm distinct from aggressive angiomyxoma. Am J Surg Pathol 1992, 16: 373-382.

2. Nielsen GP, Rosenberg AE, Young RH, Dickersin GR, Clement PB, Scully RE. Angiomyofibroblastoma of the vulva and vagina. Mod Pathol 1996, 9: 284-291.

3. Gokdemir G, Baksu B, Baksu A, Davas I, Koslu A. Features of patients with vulvar dermatomes in dermatologic and gynecologic practice in Turkey: is there a need for an interdisciplinary approach?. J Obstet Gynecol Res. Oct 2005; 31(5): 427-31. [Medline].

4. Hsu IH, Chang TC, Wu CT, Chen RJ, Chow SN. Angiomyofibroblastoma of the vulva. Journal of the Formosan Medical Association.2004; 103(6): 467-471. [PubMed].

5. Ito M, Yamaoka H, Sano K, Hotchi M. Angiomyofibroblastoma of the male inguinal region. Arch Pathol Lab Med 2000, 124ss.

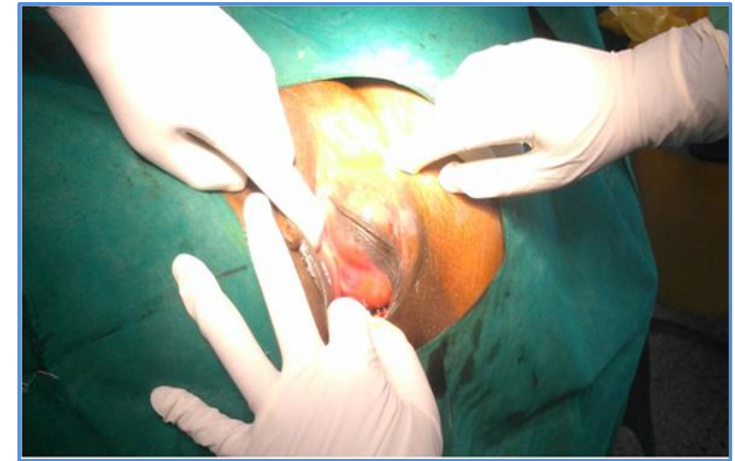

Fig. 1: Clinical appearance

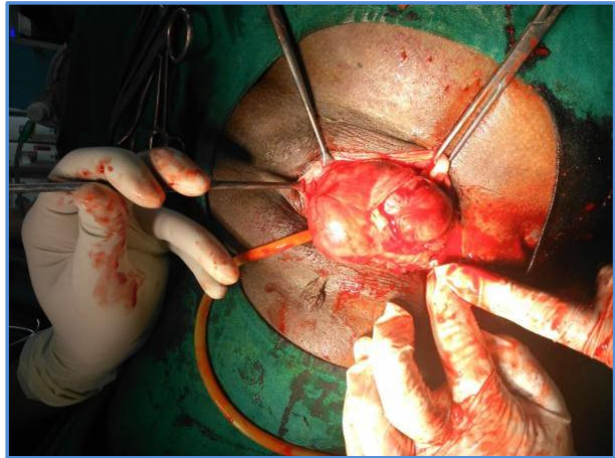

Fig. 2: Per operative - note the non-encapsulated lobulated

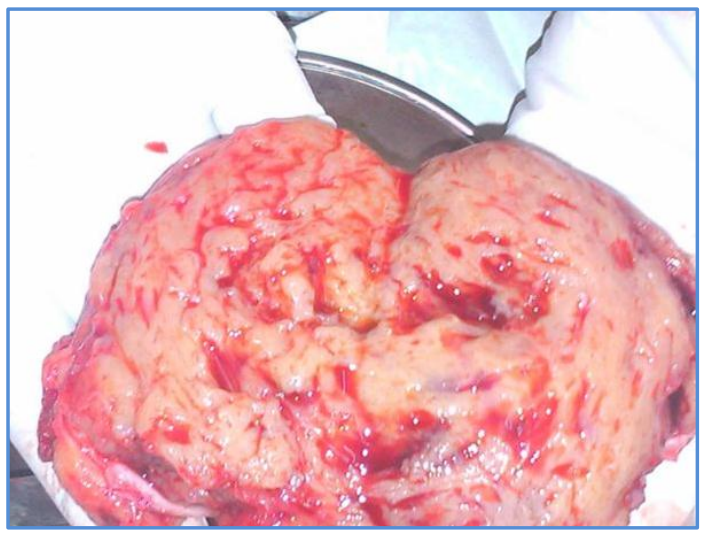

Fig. 3: Cut section - fatty tissue with multiple blood filled cystic spaces 


\section{CASE REPORT}

\section{AUTHORS:}

1. R. C. Prameela

2. Anusha K. S.

3. Akhila M.V.

\section{PARTICULARS OF CONTRIBUTORS:}

1. Associate Professor, Department of Obstetrics and Gynaecology, Cheluvamba Hospital, Mysore Medical College and Research Institute.

2. Post Graduate student, Department of Obstetrics and Gynaecology, Cheluvamba Hospital, Mysore Medical College and Research Institute.

3. Post Graduate student, Department of Obstetrics and Gynaecology, Cheluvamba Hospital, Mysore Medical College and Research Institute.

\section{NAME ADDRESS EMAIL ID OF THE CORRESPONDING AUTHOR:}

Dr. R. C. Prameela, $4544,16^{\text {th }}$ Main,

Vijayanagar $2^{\text {nd }}$ Stage,

Mysore-570017.

Email: rcprameela@yahoo.com

Date of Submission: 01/09/2014.

Date of Peer Review: 02/09/2014.

Date of Acceptance: 11/09/2014.

Date of Publishing: 18/09/2014. 